\title{
Comparative Study of Wireless Technologies Wi-Fi and Wi-MAX
}

\author{
Hasambiyev I.V. \\ Head of Department of Communication Networks \\ and Switching Systems \\ Grozny State Oil Technical University named after M.D. \\ Millionshikova \\ Grozny, Russia \\ hiv_77@mail.ru

\section{Daudov I. M.} \\ Department of Programming and IT technologies» \\ Chechen State University \\ Grozny, Russia \\ Ibr024@mail.ru
}

\begin{abstract}
The article presents the comparison analysis of wireless technologies Wi-Fi and Wi-MAX. Today, these technologies are key in modern society, no organization can do without these technologists. The aim of the scientific article is to identify the most popular and sought-after wireless technology, to determine the general characteristics of $\mathrm{Wi}-\mathrm{Fi}$ and $\mathrm{Wi}-\mathrm{MAX}$ and their main differences. Also, the scope of each of the technologies and the possibility of $\mathrm{Wi}-\mathrm{Fi}$ and Wi-MAX collaboration are considered, mutually complementing each other, which greatly increases the scope of their application.
\end{abstract}

Keywords-technologies; Wi-Fi; Wi-MAX; wireless technologies; DSL-connections; data transmission; broad-leaved technologies; established connections.

\section{INTRODUCTION}

With the development of IT technology, more and more new types of wireless technologies are emerging. This article discusses the most relevant of them, namely Wi-Fi and WiMAX. These technologies are necessary in every organization, educational institution, etc. After conducting a comparative analysis, the authors identify which of these two wireless technologies is more widely used, and why most use this technology. Speaking of wireless networks, everyone may have heard that the term Wi-MAX is increasingly being used as a technology that will replace Wi-Fi. If you are wondering what the differences between these two are, then this article is intended to answer your questions accurately [1].

The first development began technology WI-FI in the 90s. It was registered in 1999. Wi-Fi is another area of our life that facilitates data transfer to different computers and reduces time. There is an organization called Wi-Fi Alliance, which owns the Wi-Fi trademark and controls its underlying technology.

\section{METHODS AND MATERIALS}

$\mathrm{Wi}-\mathrm{Fi}$ is a wireless LAN technology that can be used in a short time. Wi-Fi is the most common wireless technology used in home, hotspots, and domestic internal wireless networks. WiFi operates at $2.4 \mathrm{GHz}$ or $5 \mathrm{GHz}$, which is an unallocated frequency band (specifically for ISM - Industrial Scientific and Medical). Wi-Fi (802.11) has several varieties, and some of them are $802.11 \mathrm{a}, 802.11 \mathrm{~b}, 802.11 \mathrm{~g}$ and $802.11 \mathrm{n} .802 .11 \mathrm{a}, \mathrm{b}, \mathrm{g}$

\author{
Khadzhiyeva L.K. \\ Department of Communication Networks and \\ Switching Systems \\ Grozny State Oil Technical University named after M.D. \\ Millionshikova \\ Grozny, Russia \\ laura.hadjieva3009@mail.ru \\ Magomedov I.A. \\ Department of Programming and IT technologies» \\ Chechen State University \\ Grozny, Russia \\ ismwork@mail.ru
}

operates at $2.4 \mathrm{GHz}$ and in the range of 40 to $140 \mathrm{~m}$ (in reality), and $802.11 \mathrm{n}$ operates at $5 \mathrm{GHz}$ with OFDM modulation technology, thus a higher speed ( $40 \mathrm{Mbps}$ with in reality) is 70 250 meters.

We can easily set up a wireless local area network (WLAN) at home using wireless routers. To gain access to Internet services, a Wi-Fi connection is established using a wireless adapter to create an access point in the immediate vicinity of the wireless router (Figure 1), which are connected to the network [2].

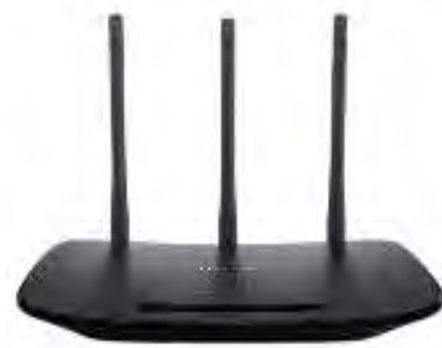

Fig. 1. Wireless router

Nowadays there is Wi-Fi almost everywhere: from houses to airports, hotels, libraries and any other place where people use wireless devices (laptops, smartphones and tablets) and computers (Figure 2).

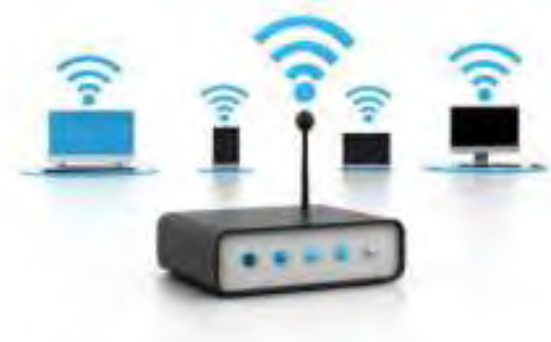

Fig. 2. Wi-Fi connection

The wireless network is broadcast over a small area, but it has no boundaries. Anyone who is near a wireless device can see that the wireless network is nearby. However, without 
knowing your password, they will not be able to connect to the network.

Here are the main benefits of setting up a wireless network:

- you can "connect" any computer / device to your network without running a cable around the house. This means that in any room in the house you can use the Internet;

- so that others cannot use your network without permission, you can configure an access password;

- it requires only a small, accessible piece of equipment, called a "router".

A distinctive feature is that you can set up a wireless network in your home quite easily and quickly.

The wireless network is broadcast over a small area, but it has no boundaries. Anyone who is near a wireless device can see that the wireless network is nearby. However, knowing your password, they will not be able to connect to the network.

However, the "wireless" function is connected to devices that can connect to the wireless network using a router.

Wi-MAX technology was developed later by Wi-Fi technology in April 2001, waiting the publication of the original IEEE 802.16 specifications. Wi-MAX is such a simple term that people tend to use it for standards, although this strictly applies only to systems that meet certain eligibility criteria established by the Wi-MAXForum. With Wi-MAX, users can really get rid of today's agreements on access to the Internet and be able to go online, wherever they are (Fig. 3.).

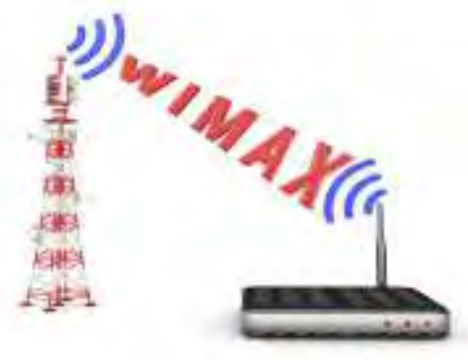

Fig. 3. Base station of Wi-MAX

It should be noted that, under the name Wi-MAX, two standards are combined: 802.16d (fixed Wi-MAX) and 802.16e (mobile Wi-MAX). The developers did not manage to combine them. Each specification has its own operating frequency ranges, bandwidth, radiation power, transmission and access methods, signal coding and modulation methods, and other characteristics. Therefore, WiMAX systems based on different versions of the IEEE 802.16 standard are practically incompatible.

The 802.16d standard (also known as 802.16-2004) uses stationary modems and PCMCIA cards for laptops. Communication is possible only with fixed subscribers. At the same time, the following characteristics can be achieved: speed up to $75 \mathrm{Mbit} / \mathrm{s}$, communication range - $25-80 \mathrm{~km}$, used frequency range - $1.5-11 \mathrm{GHz}(3.5$ and $5 \mathrm{GHz}$ are used more often).

The 802.16e standard (also known as 802.16-2005) is optimized for working with mobile users. The characteristics are as follows: speed up to $40 \mathrm{Mbit} / \mathrm{s}$, communication range $1-5 \mathrm{~km}$, used frequency range - 2.3-13.6 GHz (most often - 2.3$2.5 ; 2.5-2.7 ; 34-3.8 \mathrm{GHz}$ ). $802.16 \mathrm{e}$ is also used to serve fixed users. It is the most often offered one to users by various providers.

Thus, mobile Wi-MAX is a competitor to modern mobile technologies, and fixed Wi-MAX to wired DSL broadband access. Final subscribers wishing to connect to Wi-MAX networks should first choose a provider and enter into an agreement. After that, they will receive a USB modem, an ExpressCard modem or a similar device. You can buy a WiMAX receiver yourself; in addition, several mobile devices and laptops already have built-in support for W-iMAX technology.

Wi-MAX can satisfy various access needs. Potential applications include expanding the possibilities of broadband access to bring them closer to subscribers, to fill gaps in cables;

- Wi-MAX can support high-bandwidth solutions needed to support the full range of multimedia services;

- Wi-MAX can help service providers cope with many of the problems they face due to increasing customer needs;

- Wi-MAX can provide broad coverage and quality of service for applications, ensuring that subscribers get the performance they expect for all types of communications;

- The Wi-MAX network can be integrated into thirdgeneration $(3 \mathrm{G})$ broadband mobile networks as well as wireless and wired networks.

Finally, Wi-MAX is intended to be the next step in the evolution of $3 \mathrm{G}$ mobile phones, using a potential combination of Wi-MAX standards called 4G.

Wi-MAX is a standard technology for providing wireless alternatives to cable and DSL-connections. However, it is also one of the ways to use Wi-Fi. Although wireless Wi-Fi devices are mainly used to connect short-range wirelessly to final subscribers' devices, such as laptops, tablets and smartphones. They are also used for connections between nodes.

Wi-Fi is more popular than Wi-MAX, so more devices have built-in Wi-Fi capabilities than Wi-MAX. However, most WiMAX implementations include hardware that allows, for example, to use the service via $\mathrm{Wi}-\mathrm{Fi}$, just as a wireless router provides the Internet for several devices.

Wi-MAX is a wireless standard for building metropolitan area networks (MAN). This is basically a wireless broadband service, an alternative to DSL [7]. This indicates a multipoint connection. The IEEE 802.16 standard can transmit data at 75 Mbps up to 3 miles. It operates in licensed LOS (LineOfSight) 10-60 GHz and unlicensed NLOS (NonLineOfSight) 2-11 Ghz. The licensed LOS range is used for the MAN network (MetropolitanArea), while the unlicensed NLOS group provides communication with final subscribers who have a range between 3-5 miles [8], as shown in Figure 9. It supports 
various radio access network topologies, which allows compatible with Wi-Fi, 3GPP and existing IP networks. It has two MAC and PHY specifications, which have different operating ranges in different countries.

Wi-MAX supports a much larger range of coverage. While a Wi-Fi signal can span a radius of several hundred feet, a fixed Wi-MAX station can span a range of up to $30 \mathrm{~km}$. Mobile WiMAX stations can broadcast up to $10 \mathrm{~km}$ (Figure 4).

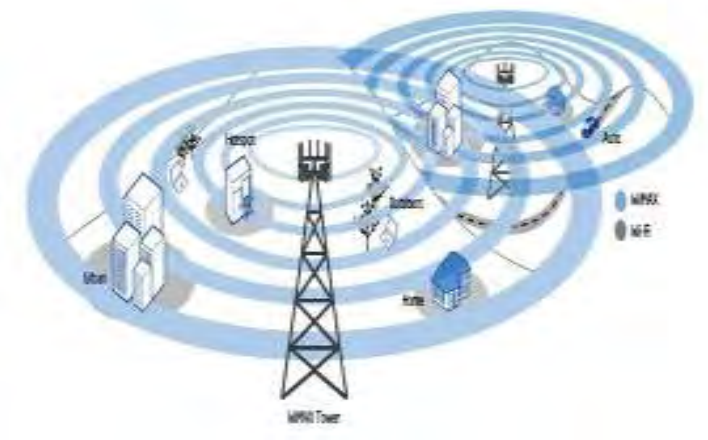

Fig. 4. Range of Wi-Fi and Wi MAX

By the way, Wi-Fi is a good wireless Internet solution for home networks, but not for large areas. To cover a large area, you need to install several Wi-Fi repeaters at regular intervals. For areas covering several kilometers, this is a rather inefficient method of providing wireless access and, as a rule, requires a large amount of maintenance. Wi-MAX, on the other hand, can cover several kilometers using one station. This greatly simplifies maintenance and provides more reliable coverage [3].

Since Wi-MAX has a large range of signals, it will potentially be used to provide access to the wireless Internet for all cities and other large areas. In fact, some Wi-MAX advocates predict that eventually it will extend Internet access to all parts of the globe.

$\mathrm{Wi}-\mathrm{Fi}$ is widely used for broadband wireless connectivity of end devices.

Wi-MAX is the right, effective wireless solution for longterm connection of several sites, such as providing access to the Internet in several houses or combining several buildings into a large connection [4].

\section{RESULTS}

Even though both technologies have similar names and WiMAX technology appeared later, it can be assumed that WiMAX is an advanced Wi-Fi model, but it is not. These technologies have various uses.

$\mathrm{Wi}-\mathrm{Fi}$ is a technology mainly designed for organizing small internal wireless networks and building wireless bridges. The Wi-MAX technology, in turn, is intended for the organization of broadband communication in the open air and for the organization of large-scale networks. Wi-MAX was developed as a city computer network (MAN). There are other differences between these technologies. Wi-MAX has better connection quality than Wi-Fi. When several users are connected to a $\mathrm{Wi}$ -
Fi access point, they literally "fight" for access to the communication channel. In turn, Wi-MAX technology provides every user with constant access. Built on Wi-MAX technology, the algorithm sets a limit on the number of users for a single access point. When a Wi-MAX base station reaches its maximum potential, it automatically redirects "redundant" users to another base station [5].

But Wi-Max is still in its initial state and will require significant investment in this infrastructure for commercial gain.

Wi-Fi is already a self-contained system, and the rapid deployment of Wi-Fi networks is not a problem now [6].

Companies with large areas may want to switch to Wi-MAX to avoid buying many repeaters required when installing a $\mathrm{Wi}$ Fi network. Now, such equipment is not wide enough in Russia [9].

As in many other areas, there is no universal technology in wireless data transmission. Wi-MAX or WI-FI is more suitable for each specific task. If the goal is to provide broadband access to the network for users, then of course Wi-MAX is suitable because this technology was originally developed for this purpose. However, if the challenge is to provide broadband access in a limited space, then WI-FI and Wi-MAX technologies are equally well suited to solve, if there is no low level of interference or interference. And for the introduction of wireless security systems or video surveillance more suitable Wi-Fi, since this direction is already quite well developed [10].

\section{CONCLUSION}

The article presents an overview of Wi-Fi and Wi-MAX wireless technologies; the authors have made the comparative analysis of wireless technology data.

The authors noted that, despite the large coverage of the territory Wi-MAX is an expensive technology. The installation of stations will take quite a bit, time and money. And it is not known if the station will fail, whether it will be repaired immediately and how long it will take [11].

Wi-Fi is one of the most popular nowadays. Even though it does not have a large area coverage, Wi-Fi technology is accessible to all at its reasonable cost and installation communication. In cases of occurrence, any problems are solved very compactly. For this and several other reasons, it is advisable to develop the Wi-Fi technology, since the pricequality ratio is proportional [12].

Thus, two wireless technologies were analyzed. According to the results of the comparative analysis, Wi-Fi has a high priority.

Undoubtedly, the advantages of Wi-MAX technology in some areas are obvious, but the difficulties did impede its further development. If Wi-MAX wants to continue development, it must be redistributed and make a reasonable plan for its positioning in the market.

Wi-MAX provides low-cost mobile Internet access and provides integrated wireless fixed and mobile services using a single on-air interface and network architecture. But the Wi-Fi spot cannot be replaced in recent years. The best development 
of Wi-MAX will be to consider how to coexist with Wi-Fi. Like the deployment image in the article, using these two technologies can provide better coverage of wireless networks. Wi-MAX has moved to a bottleneck that could not be avoided. As mobile operators gradually move to the LTE market, WiMAX had to face enormous problems. At the same time, it gave Wi-Fi a lot of space to continue development. Undoubtedly, Wi-MAX has advantages in some areas, but still risks being acquired. Wi-MAX should determine its location, find an appropriate market development and think about better coexistence with Wi-Fi.

\section{Acknowledgements}

The authors express to Prof. E. D. Alisultanova, Head of Department of Computer Science of Grozny State Oil Technical University named after M.D. Millionshikova, thanks and deep appreciation for advice and valuable comments in preparing this article.

\section{References}

[1] E.V. Smirnova, E.A. Romanova, A.V. Proletarskiy, Technology of modern wireless networks Wi-Fi, Moscow: MGTU im. N.E. Baumana, 2017.

[2] V. Vishnevskiy, S. Portnoy, I. Shakhnovich, (eds), Encyclopedia of WiMax. 4G path, Moscow: Tekhnosfera, 2012.
[3] V.G. Olifer, N.A. Olifer, (eds), Computer networks. Principles, technologies, protocols. Textbook, Saint-Petersburg; Piter, 2014.

[4] P. Roshan, The Basics of Building 802.11 Wireless Local Area Networks, Moscow, Vilyams, 2004.

[5] Ya.S. Dymarskiy, N.P. Krutyakova, G.G. Yanovskiy, eds., (2003) Network management: principles, protocols, application tasks, Moscow, ITTS Mobil'nyye kommunikatsii.

[6] A.V. Proletarskiy, I.V. Baskakov, R.A. Fedotov, D.N. Chirkov, eds., Wireless network, Moscow: Publishing MSTU. N. E. Bauman, 2010.

[7] S.J. Vaughan-Nichols, Achieving wireless broadband with WiMax, IEEE computer, 37, pp. 10-13, 2004.

[8] S. Dekleva, J. Shim, U. Varshney, G. Knoerzer, Evolution and emerging issues in mobile wireless networks. Communications of the ACM, Vol. 50, pp. 38-43, 2007.

[9] COMPARATIVE ANALYSIS OF WIRELESS TECHNOLOGIES FOR INTERNET-OF-THINGS BASED SMART FARM. Retrieved from: https://www.researchgate.net/publication/317425702_COMPARATIVE ANALYSIS OF WIRELESS TECHNOLOGIES FOR INTERNETOF-THINGS_BASED_SMART_FARM

[10] Jonh Ross, The book of Wi-Fi Install, Configure, and Use $802.11 \mathrm{~b}$ Wireless Networking, p. 312, 2005.

[11] Y. Fan, Z. Huaibei, Z. Lan, F. Jin, Improved security scheme in WMAN networks based on the IEEE 802.16 standard.

[12] D. Johnston, J. Walker, Security Overview in IEEE 802.16. IEEE Security and Privacy, vol. 2, pp. 40-48.

[13] A. Furuskar, Allocation of multiple services in multi- access wireless networks, Mobile and wireless communication networks, pp.201-205, 2002 . 